\title{
INTERACTION BETWEEN VARIOUS TERRAIN PORTRAYALS AND GUIDANCE/TUNNEL SYMBOLOGY CONCEPTS FOR GENERAL AVIATION SYNTHETIC VISION DISPLAYS DURING A LOW EN-ROUTE SCENARIO
}

\author{
M.A. Takallu, Lockheed Martin, Hampton, Virginia \\ D.T. Wong, A.P. Bartolone, M.F. Hughes, and L.J. Glaab, NASA Langley Research Center, Hampton, \\ Virginia
}

\begin{abstract}
In support of the NASA Aviation Safety Program's Synthetic Vision Systems (SVS) Project, a series of piloted simulations were conducted to explore and quantify the relationship between candidate terrain portrayal concepts and guidance/tunnel symbology concepts, specific to General Aviation (GA).
\end{abstract}

The experiments were conducted in a fixed based flight simulator equipped with two separate 6-inch Liquid Crystal Display (LCD) Head Down Displays, one serving as a glass cockpit style Primary Flight Display (PFD) and the other as a Navigation Display (ND). This work is the second part of a three-part study related to the Symbology Development for Head Down Displays (SD-HDD) test series. The focus of this experiment was on advanced low altitude en route maneuvers simulating a transition into Instrument Metrological Conditions (IMC) in the central mountains of Alaska (Merrill Pass). A total of $18 \mathrm{GA}$ pilots, with three levels of pilot experience, evaluated a test matrix of four terrain portrayal concepts (TPC) and six guidance/tunnel symbology (GSC) concepts.

Both quantitative and qualitative measures were recorded and analyzed. Quantitative measures included all pilot/aircraft performance data, flight technical errors (FTE), flight control inputs, and selected physiological data. The qualitative measures included pilot comments and pilot responses to the structured questionnaires such as perceived workload, subjective Situation Awareness (SA), pilot preferences, and the rare event recognition. Only a sample of the results of FTE, SA and workload is reported here.

There were statistically significant effects found from GSC and TPC but no significant interactions between TPCs and GSCs for this experiment. Lower FTE and increased SA were achieved using SVS displays, as compared to the baseline Pitch/Roll Flight Director (PRFD) and Blue Sky Brown Ground (BSBG) combination. These results indicate that all pilots performed very well, mostly within the $75 \mathrm{ft}$ of vertical and lateral limits indicated by one dot of the course deviation indicators. With the same SVS training provided to all three groups, low time VFR pilots performed as well as IFR pilots in low altitude en-route scenario with IMC. Overall those GSCs that have both Guidance Cue and Tunnel performed better than the other concepts.

\section{Introduction}

Limited visibility is the single most critical factor affecting both the safety and capacity of worldwide aviation operations. With the integration of Global Positioning Systems (GPS), advanced solid state Attitude and Heading Reference Systems (AHRS), and the imagery derived from terrain, obstacle, and airport databases, into a Primary Flight Display (PFD), Synthetic Vision Systems (SVS) displays provide the pilot with an unobstructed view of the outside world, regardless of weather conditions and time of day. In addition, through the integration of advanced symbology (i.e. highway in the sky, velocity vectors, etc.), pilot situation awareness and navigation performance is drastically improved with no effective increase in pilot workload. Recently, there have been many studies dealing with various components of SVS and the merit of SVS as a whole [1-8]. However, the proper marriage of all these new technologies into the PFD has a profound implication to the future of the safety and security of aviation, and it is of utmost interest to the NASA, FAA, and the aircraft/avionics manufacturers [9].

A goal of the Synthetic Vision Systems (SVS) Project of the NASA Aviation Safety and Security 
Program is to eliminate poor visibility as a causal factor in aircraft accidents as well as enhance operational capabilities through application of SVS technology. SVS displays can help to enhance pilot's SA, radically reducing the occurrence of Controlled Flight into Terrain (CFIT) accidents. In addition, SVS displays have the potential to reduce or eliminate pilots' spatial disorientation (SD), a primary cause of Low Visibility Loss of Control (LVLOC) accidents.

As an initial investigation, the SVS-GA team conducted a simulation study that focused on determining the associated benefits of SVS displays towards reducing LVLOC and CFIT accidents for GA pilots [7-8]. The study simulated an inadvertent IMC encounter during basic air maneuvers and was conducted in the General Aviation Work Station (GAWS) at the NASA Langley Research Center (LaRC). Results of those simulations demonstrated the effectiveness of generic SVS displays as compared to conventional $\mathrm{GA}$ round dials in reducing pilot errors and thus improving pilot's ability to control the aircraft during IMC.

The next series of experiments were conducted in the GAWS and in the Langley Cessna 206 (single engine) aircraft. These experiments focused on issues of Terrain Portrayals for Head Down Displays (TP-HDD). The TP-HDD experiments concentrated on core technology issues such as the effect of terrain texturing, Digital Elevation Model (DEM) resolution, and the size of the Field of View (FOV) on pilot performance and Situation Awareness (SA). Variations of symbology were 1) conventional round dials with a pair of Course Deviation Indicators (CDI); 2) baseline PFD with a simple tunnel (no-terrain depicted on PFD); and 3) SVS with the same tunnel as in 2). The researchers were able to demonstrate the efficacy of SVS displays for a comprehensive spectrum of pilots in both mountainous and flat-maritime environments.

When SVS terrain was integrated with simple tunnel guidance, it improved both inexperienced and experienced pilot performance versus the baseline round dials conditions. When terrain was added to the PFD, situation awareness was drastically enhanced and the mental workload was decreased with no degradation in EP performance. Pilots preferred higher-resolution DEMs. Based on pilot preferences evaluations 3 arc-sec was considered satisfactory and 30 arc-sec, while least preferred of the 3 DEMs, was still considered a great enhancement over standard gauges. Among the three FOVs $(30,60$, and 90$)$ studied, $60^{\circ}$ fieldof-view was the most useful and lower field-ofview use was only possible in calm conditions (during the flight tests in the Cessna 206).

\section{Objective of Experiment}

Based on the above findings and NASA's support of the FAA Capstone Project a follow on series of experiments were designed to address many issues related to the Symology Development for Head Down Displays (SD-HDD) for SVS. The SD-HDD simulations were conducted to explore and quantify the relationship between candidate terrain portrayal concepts (TPC) and guidance/tunnel symbology concepts (GSC).

The objectives of the three parts SD-HDD experiments were to: 1) Establish interactions between GSCs and TPCs on a Primary Flight Display (PFD) for VMC-like operations (Approach, Missed Approach, and complex mountain pass maneuvers) in both an IMC and terrain-challenged environments, 2) Develop recommendations for SVS-GA symbology and terrain texture on a PFD, 3) Demonstrate application of SVS technology for advanced operational procedures, and 4) Evaluate altitude and range estimations for different terrain texturing methods and minification factors.

The first experiment in the series studied the terminal area operations with two challenging scenarios, simulating a curved approach and a curved go-around scenario, at Juneau, Alaska. Discussion of the results of this experiment can be found in reference [13]. The second and the third experiments of the SD-HDD concentrated on low altitude en route maneuvers in the central mountains of Alaska (Merrill Pass). The second experiment is the focus of this paper.

\section{Method}

Three independent variables were selected to address the experiment objectives. The independent variables were the TPC with 4 levels, the GSC with 6 levels, and EP qualifications (Rating) with 3 levels. All these 72 combinations 
were repeated randomly with one replicate (i.e. two evaluations) for each EP. Therefore each EP participated in a total of 144 runs of 5 minutes duration over a period of two days.

On the second half of the second day of the experiment, a seventh variation of the GSC was presented to each EP that had No Guidance Cue and No Tunnel (NGC/NT). A rare event scenario was also conducted as the last run of the second day. The discussion and presentation of the results of both the rare event scenario and the NGC/NT runs will be presented in subsequent documents.

\section{GAWS Set-Up}

The experiments were conducted in the General Aviation Work Station (GAWS) at Langley Research Center, a fixed based flight simulator equipped with two separate 6-inch LCD Head down Displays (HDD). The two 6-inch displays were Commercially-Of-The-Shelve (COTS) VGA monitors. The left 6-inch display served as the SVS-PFD and the right one as a navigation display (ND) with multi-level range selection capability, developed at NASA LaRC. Pilot selectable fields of view (FOV) (horizontal angle of the image that is presented on the display) of 30 and 60 degrees were available throughout the scenarios for the PFD and the boundaries were depicted on the ND. A SXGA overhead projector provided the Out-The-Window (OTW) view depiction.

The hardware center piece was the Precision Flight Controls PC-based Aviation Training Device (PFC-PCATD), model PI-142 instrument trainer, consisting of dual yoke and rudder pedals with a radio stack positioned between the two pilot seats. The evaluation pilots flew the scenarios from the left seat. The right seat was occupied by the instructor during the training sessions only. The flight simulator software was configured with a Cessna C-172 dynamic flight model from Initiative Computing Electronic Instrument Training Environment (ELITE) Simulation Solutions Company.

\section{Terrain Portrayal Concepts}

The distance between elevation data points (post-spacing) for a given a given database is called Digital Elevation Model (DEM) resolution. The highest terrain resolution available ( 2 arc-sec) was selected for the Merrill Pass simulations. Due to convergence of the longitudes nearing the North Pole, this is almost equivalent to the 1 arc-sec (30 $/ 98 \mathrm{ft}$ post-spacing) DEM option investigated during the TP-HDD study simulating Southern Virginia areas.

Terrain-texturing refers to the method used to color the polygons that comprise the SVS terrain database. The three primary SVS texturing concepts tested were constant-color (CC), elevation-based generic (EBG), and photo-realistic (PR). The cultural features, such as roads and rivers, were included as objects in the SVS terrain database. A standard generic Blue Sky/Brown Ground (BSBG) PFD served as the baseline TPC, Figure 1. Of course, there were no cultural features included in BSBG.
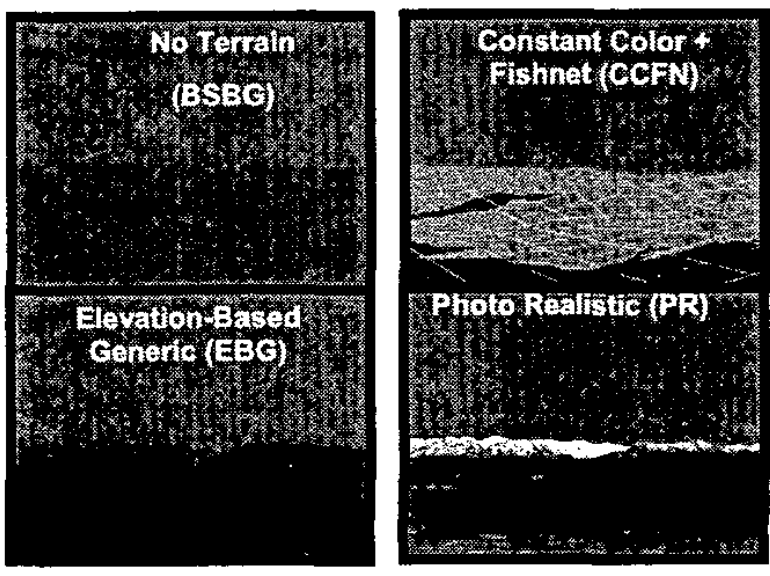

Figure 1. Terrain Portrayal Concepts (TPC)

The $\mathrm{CC}$ texturing concept was developed to represent a current industry concept. The fishnet had $500 \mathrm{ft}$ squares.

The EBG texturing concept consists of twelve equal-height coloring bands that correspond to different absolute terrain elevation levels, similar to the colors employed for Visual Flight Rules (VFR) sectional charts. Lower terrain levels are colored with darker colors; higher terrain levels are assigned lighter colors. A shade of green was set to the sea level elevation. The lightest color was set to the highest terrain within a rectangle of $157 \times 180$ $\mathrm{nm}$ surrounding Merrill Pass, approximately 9,000 $\mathrm{ft}$ MSL.

The PR texturing concept was derived from full color ortho-rectified $4 \mathrm{~m}$ satellite imagery data. 
The resulting scene was a highly realistic view due to the photographic imagery employed. The PR texturing required special graphics hardware due to the amount of texture memory required to create the realistic scene. Figure 1 illustrates the four TPCs tested in this experiment.

\section{Guidance/Tunnel Symbology Concepts}

As employed for this experiment, Highway-inthe-Sky (HITS) concepts consist of two main elements. The first element is a Three Dimensional (3-D) pathway of some type and the second feature is referred to as a Guidance Cue (GC). One of the most common depictions of a pathway is a tunnel. The tunnels are a perspective view of the oncoming boxes. Common variations of tunnels are the shape, size, color, whether the comers are connected, and whether the boxes are connected along the flight path, etc.

Each pathway-based GSC employed for this effort represents significant guidance symbology designs developed and used in commercial products as well as other mature pathway-based GSCs. The approach for this study was to employ each GSC as developed and not refine each concept to preserve specific independent parameters (such as tunnel size, color, etc.). The primary independent variable affected by GSC was visual complexity from simple PRFD command bars up to connected box tunnels with sliding rail guidance cues and lateral predictor.

In addition to the tunnels depicting the lateral and vertical limits around the path, path based CDIs were also implemented. The limits of the CDIs were set to $75 \mathrm{ft}$ per dot with $150 \mathrm{ft}$ ( 2 dots) limits for a full deflection in both horizontal and vertical directions from the flight path. The CDI scale remained constant for every GSC studied in this experiment. The guidance cues selected were based on currently available technology and were 1) a split cue pitch/roll flight director; 2) a single cue ghost plane; and 3) a single cue guidance box. There were three possible combinations of GCs and Tunnels as shown below:

A- Guidance Cue, No Tunnel

1- Pitch/Roll Flight Director (PRFD)

2- Ghost Plane (GP)
B- No Guidance Cue, With Tunnel:

\section{3- Unconnected-Boxes Tunnel (UBT)}

4- Crows Feet Tunnel (CFT)

C- Guidance Cue, With Tunnel:

5- Crows Feet Tunnel \& Ghost Plane (CFGP)

6- Connected Boxes Tunnel \& Guidance Square (CBT)

Each one of GSCs are described in detail below and illustrated in Figures 2 through 7 . A discussion of the basic non-guidance related PFD symbology used here can be found in reference [12].

\section{1-GSC: Pitch/Roll Flight Director (PRFD)}

For the PRFD, the displacement of the horizontal and vertical error bars (magenta in color) from the water marker indicate the commands for pitch and roll, respectively, to correct for path errors. To achieve a precise path control (to center the CDIs), EPs were instructed to position the water-marker on the horizontal and vertical bars of PFRD thus bring the bars to a cross-hair shape (see Figure 2).

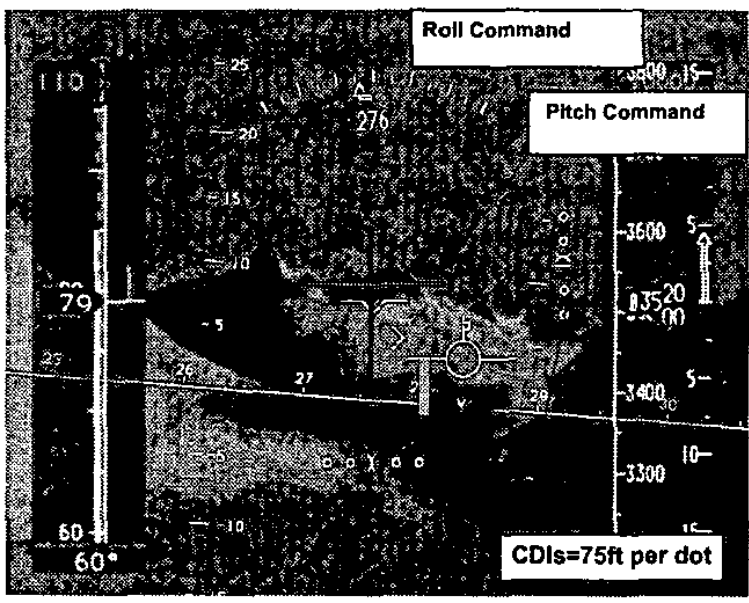

Figure 2. Pitch/Roll Flight Director

\section{2- GSC: Ghost Plane}

In this concept, there is a symbolic aircraft depicted on PFD moving 5 seconds ahead of own ship. Once own ship moves off the desired flight path the GP shows the correction path necessary to get back on the desired flight path. To achieve a precise path control (to center the CDIs), EPs were instructed 
to position the path vector (also known as velocity vector) on the ghost plane. In extreme cases where the pilot is far off course, the GP remains pegged at the corner of viewing area, changes color to amber, and a line is drown from velocity vector to the GP indicating the direction of the required correction. This resembles a no-slack tow rope connecting a sailplane to the tow plane (see Figure 3).

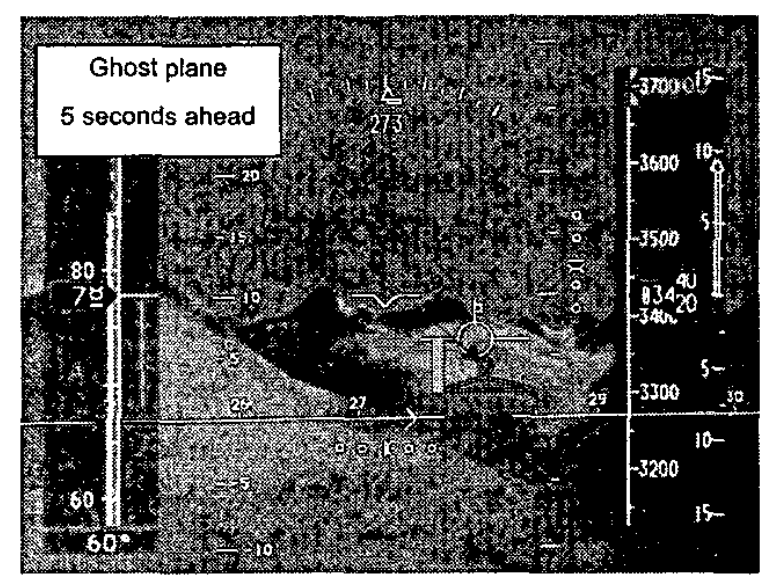

Figure 3. Ghost/Follow-me Plane

\section{3-SGC: Unconnected Boxes Tunnel (UBT)}

This concept is very similar to the industry concept which was used in the FAA's Capstone-2 Project. The corners of the green boxes are connected but the boxes are not connected to each other. Each tunnel box is $400 \mathrm{ft}$ wide and $320 \mathrm{ft}$ tall. There is no guidance cue for this GSC. For precise flight path control, the EPs were instructed to position the velocity vector within the oncoming boxes such that it would lead to centered CDIs (see Figure 4).

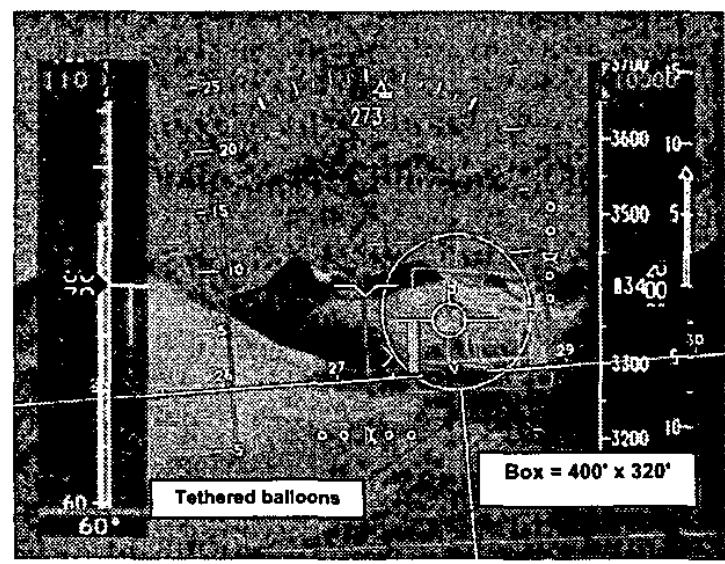

Figure 4. Unconnected Connected Boxes

\section{4-GSC: Crow-Feet tunnel}

This tunnel was developed at LaRC in conjunction with the Ghost Plane concept. The corners of the magenta boxes are not connected and are shaped in three dimensions resembling a crowfoot. The CFT dimensions were kept as $600 \mathrm{ft}$ wide and $350 \mathrm{ft}$ tall. The EPs were instructed to place the velocity vector in the oncoming boxes to center the CDIs (see Figure 5).

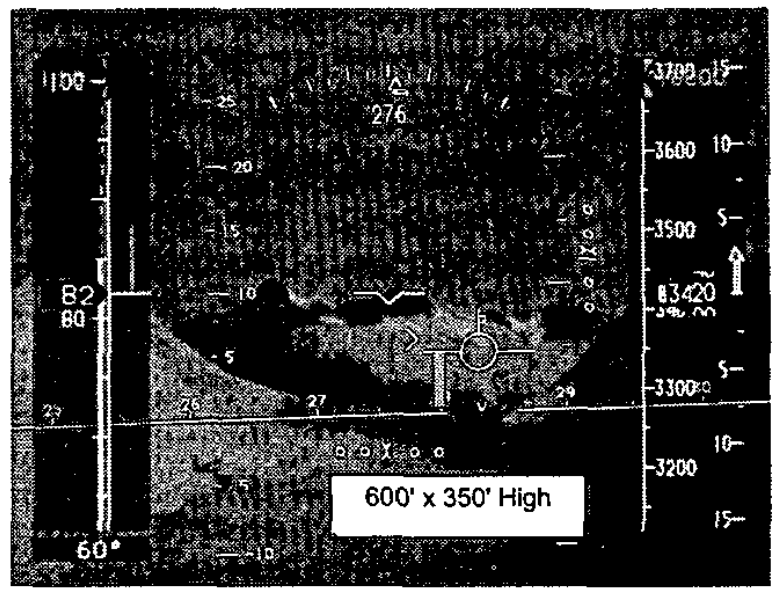

Figure 5. CrowsFeet Tunnel

\section{5-GSC: Connected Boxes Tunnel \& Guidance Square}

This GSC is based on another industry concept where the white boxes are connected to each other. In addition to the $300 \mathrm{ft}$ by $300 \mathrm{ft}$ tunnel, there is a magenta guidance square moving 5 seconds ahead of ownship providing the guidance cue. As an integral part of this concept, the velocity vector has a quickening of 5 seconds in the lateral direction thus it is referred to as path predictor or velocity predictor. For precise flight path control EPs were instructed to use the tunnel and position the predictor in the center of the guidance square (see Figure 6). 


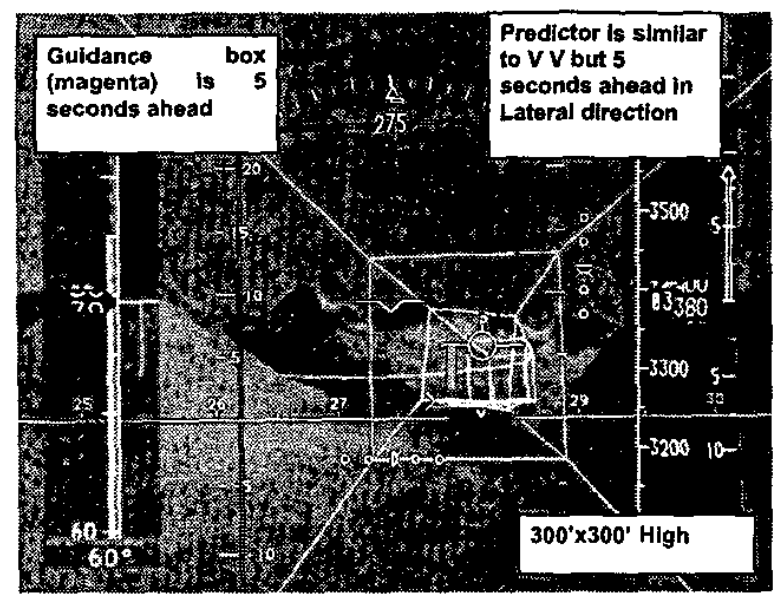

Figure 6. Connected Boxes Tunnel

\section{6- GSC: Crow Feet Tunnel \& Ghost Plane}

This GSC is a combination of the GSC \#2 and GSC \#4 and (as mentioned) developed at LaRC. The EPs were instructed to follow the tunnel and position the velocity vector on the GP for precise flight path control (see Figure 7).

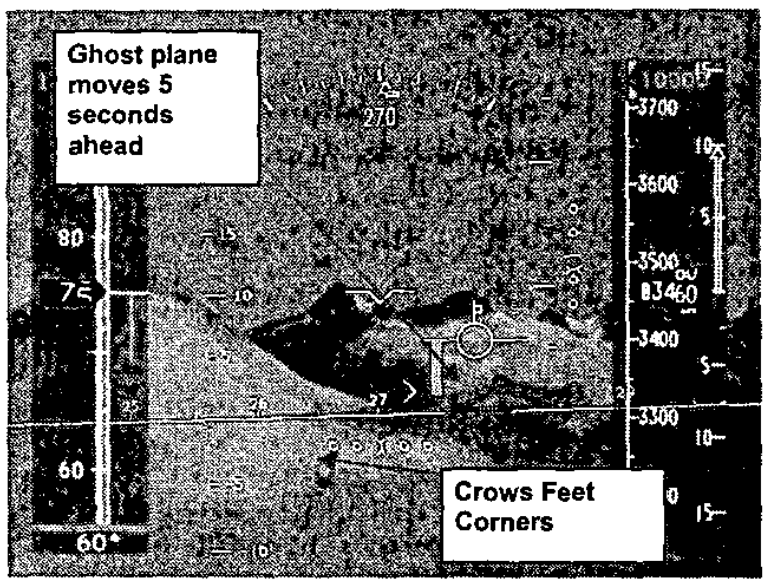

Figure 7. CrowsFeet Tunnel \& Ghost Plane

\section{Evaluation Pilots}

Three groups of Evaluation Pilots (EPs), a total number of 18 , were recruited from around the country representing typical General Aviation (GA) pilot spectrum. The first group of EPs (VFR group) consisted of 6 low-time pilots, each with less than 400 hours of Total Time (TT) flight experience and no instrument training beyond that required for the FAA private pilot's license (mean TT $=175$ hours; mean age $=44$ years). The second group (IFR) consisted of pilots with less than 1000 hours of TT and with an Instrument Rating (mean TT $=450$ hours; mean age $=38$ years). The third group of EPs (HIFR) consisted of professional test pilots from NASA, Boeing, FAA, and Alaska commercial operators with several thousands of hours TT each (mean TT $=8574$ hours; mean age $=56$ years).

\section{Training of Evaluation Pilots}

Before the start of the experiment, each pilot received an extensive pilot briefing (Ground School), as well as approximately one-hour of simulator training in the GAWS with a FAA certified flight instructor for instruments (CFII). The objective the briefing and the training were to familiarize each EP with the objectives of the experiment and educate the subjects on the salient features of the symbology and simulator functionality. FAA/Jeppesen-style training syllabus was utilized for training and to test EPs skills according to the FAA Practical Test Standard (PTS) for Private Pilot License (PPL). EPs were instructed to 1) Use all display information to minimize pilot flight technical errors; 2) Avoid hazardous terrain or flight situations; and 3) Communicate their intentions and take corrective action when encountering hazardous situations.

\section{Experiment Scenario}

In Alaska, many populated areas are surrounded by high mountainous terrain and are accessible only by air or sea. This has generated a high-reliance of GA aircraft as the main mode of transportation. Frequent low altitude icing levels exist in this region, and when combined with no usable IFR infrastructure, it makes traditional IFR operations impossible. Pilots are forced to navigate through treacherous mountain passes, sometimes in very poor visibility, in order to complete their flights. Thus central mountains of Alaska were a natural choice for a challenging low altitude en route scenario. At Merrill pass, the terrain rises towards the pass requiring the pilot to recognize the upslope from terrain depiction to climb through the pass. After passing through the crest of the mountain the terrain slopes down towards the glaciers. Maintaining path position was very essential in maintaining adequate terrain clearance. 
The scenario started with the aircraft flying at $500 \mathrm{ft} \mathrm{AGL}$ and Indicated Airspeed (IAS) of 100kt in VMC (see Figure 8). A transition to IMC was simulated at one minute into the scenario. The EPs task was to maintain 500 AGL which could be an optimal altitude for search and rescue operations.

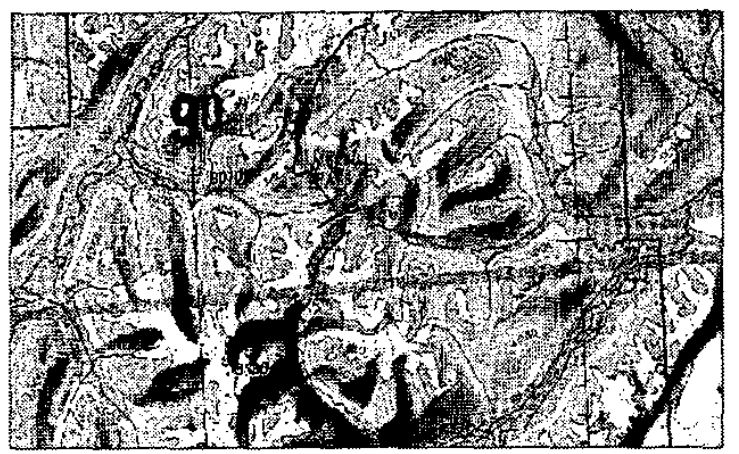

Figure 8. Experiment Scenario, Merrill Pass, AK

\section{Dependent Variables}

The dependent variables were both objective and subjective measures. The objective data measures included vertical and lateral path error (FTE), percent of time the pilot was within $+/-75 \mathrm{ft}$ of the center of the path laterally and vertically, pilot control inputs, and pilot/aircraft performance data.

The subjective data measures included a 3-D Situation Awareness Rating Technique (SART), a standard NASA-TLX (TLX) workload index, and specific questions about terrain awareness and guidance awareness.

\section{Test Protocol}

Each EP participated in two 8-hour days of testing, conducting 60 runs of 5-minute duration. At the end of each data collection run the subject pilot was required to complete a three-page subjective run questionnaire, administered digitally on a Tablet PC. The run questionnaires soliciting the EP's subjective estimates of SA (SART), perceived workload (NASA-TLX), and handling qualities (Cooper Harper) for each one of the 144 $(4 \mathrm{TPC} \times 6 \mathrm{GSC} \times 2$ Replicates $=144)$ displays immediately after exposure to each display. The schedule followed was: Day 1; Pilot Briefing, Overall Training, Data Collection for all Guidance/Tunnel Concepts, Day 2; Data Collection continued in the morning. In the afternoon, data collection continued for NGC/NT runs (w/Physiological Data) and finished with the rare event scenario. During the exit interview several sets of questionnaires, related to EP preferences and $\mathrm{SA}$, were administration.

\section{Results}

Time history data was analyzed in order to generate minimum, maximum, Root Mean Square (RMS), Standard Deviation (StD) statistical parameters. Computations were performed for a constant distance of $6.38 \mathrm{~nm}(1.92-8.3)$ which corresponds to a waypoint near the start of IMC phase of flight to a waypoint near the end of scenario (right after clearing the Pass). Then both the above objective data and subjective measures (from the questionnaires) were statistically analyzed using SPSS software. Univariate Analyses of Variances (ANOVA) and Post Hoc analyses of all measures were performed. The results discussed here will be limited to the RMS of the FTEs (lateral and vertical path errors) and the SART and TLX results and they are organized according to the independent variables (TPC, GSC, and Rating) and their interactions.

\section{Effect of TPC}

The particular type of terrain displayed on the PFD affected both pilot objective and subjective measures. The RMS of the Lateral Path Deviation (LPD) showed a statistically significant effect of TPC, $F(3,720)=5.009(P<.05)$, creating several subgroups. The TPCs creating the best LPD were $E B G$ and $P R$. The CCFN and BSBG belonged in the other subgroup. Figure 9 illustrates the bar charts of the mean RMS of LPD. It can be seen that the LPDs were approximately $8 \mathrm{ft}$ lower for PR and EBG than CCFN and BSBG. 


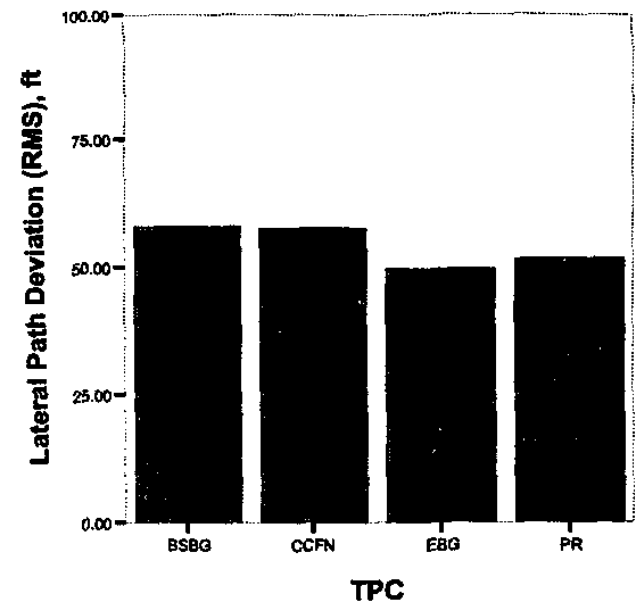

Figure 9. Mean RMS of Lateral Path Deviation

The RMS of Vertical Path Deviation (VPD) showed a statistically significant effect of TPC, $\mathrm{F}(3,720)=4.24(\mathrm{P}<.05)$, creating two subgroups. VPD was statistically lower for EBG than the other 3 TPCs (PR, CCFN, and BSBG) with a mean of 25.5 for EBG versus 28.5, 30.1, and 30.2 for PR, CCFN, and BSBG, respectively. While the difference in VPD between EBG and the other TPCS may not be operationally significant, reductions in VPD can indicate an increased amount of SA, or decreased workload, resulting from facilitated pilot scan patterns for the EBG TPC (see Figure 10). The EBG TPC provides terrain in a somewhat coded yet highly intuitive manner providing the pilot with essential information, such as where is the high terrain, and eliminating secondary information, such as whether the terrain is rock or dirt.

Effect of TPC on SA is shown by the SART data in Figure 11. The statistically significant results, $F(3,720)=31.5(p<.05)$, show that there were 3 subgroups formed. BSBG had the lowest $\mathrm{SA}$; CCFN was in the middle; and EBG and PR providing the highest levels of SA. Here the value of perceived SA for EBG and PR was almost twice the value for the other two TPCs (see Figure 11).

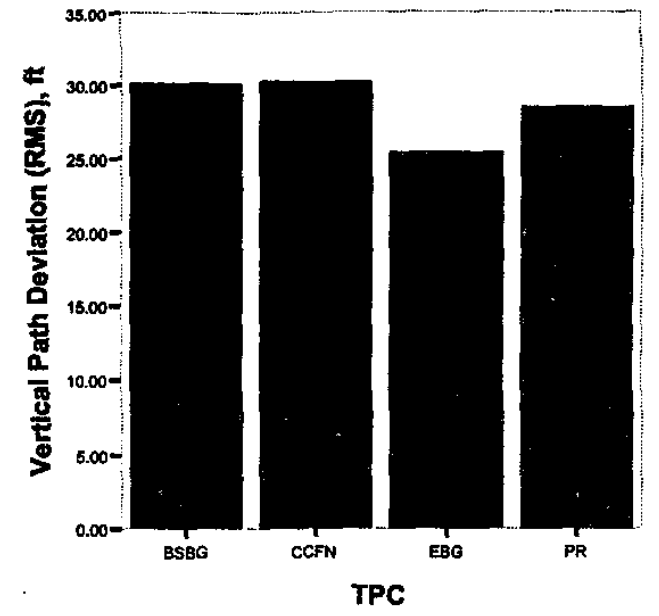

Figure 10. Mean RMS of Vertical Path Deviation

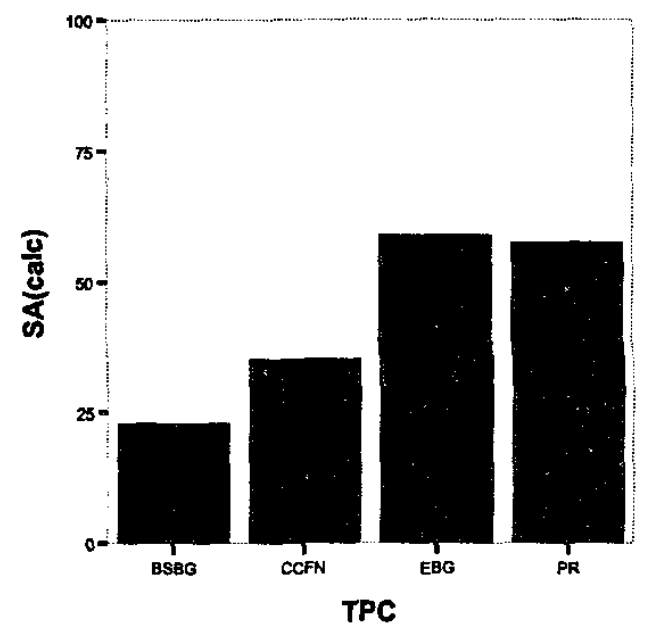

Figure 11. Perceived Situation Awareness, SART

The results of subjective workload, as indicated by TLX data, are provided in Figure 12. The effect of TPC on TLX data was statistically significant, $F(3,720)=12.226(p<.05)$, with two subgroups emerging from the post-hoc analyses. EBG and PR provided the lowest perceived workload with CCFN and BSBG being in the other group. 


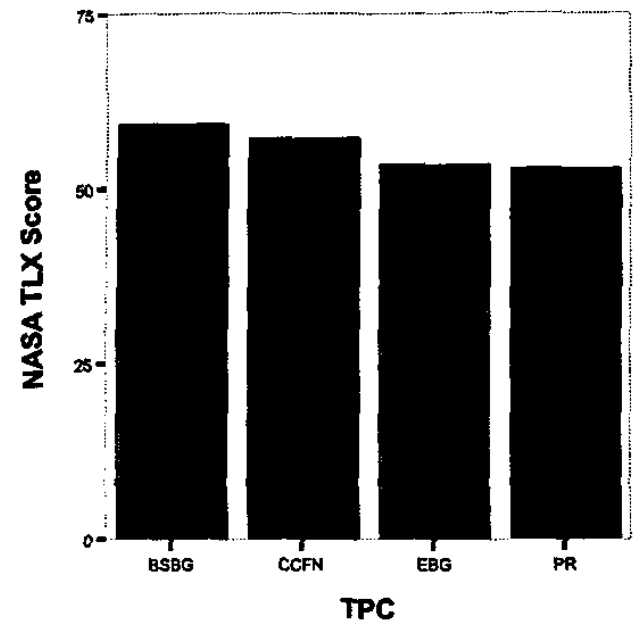

Figure 12. Perceived Workload, TLX

\section{Effect of GSC}

LPD results for GSC are presented in Figure 13. ANOVA results for these data $(F(5,720)$ $=108.12(\mathrm{p}<.05)$ indicate that the CBT, CFTGP, and GP GSCs were in the first subset, UBT in the second, and CFT and PRFD providing the worst performance. It should be noted that the best performing GSCs (CBT, CFTGP, and GP) provide LPDs that were approximately one half of the worst group (PRFD, and CFT). The magnitude of this result is operationally significant and can greatly facilitate new types of operations requiring low FTE.

Mean RMS values of VPD for GSC are provided in Figure 14. The effect of GSC on VPD was statistically significant, $F(5,720)=105.48$ $(p<.05)$, with post-hoc analysis generating five subgroups. The CFTGP and GP generated the lowest VPD, with CBT being the next lowest followed by UBT, PRFD and finally CFT, in that order. Similar to LPD, VPD results for the best group (CFTGP and GP) were less than one half of the worst subset (CFT).

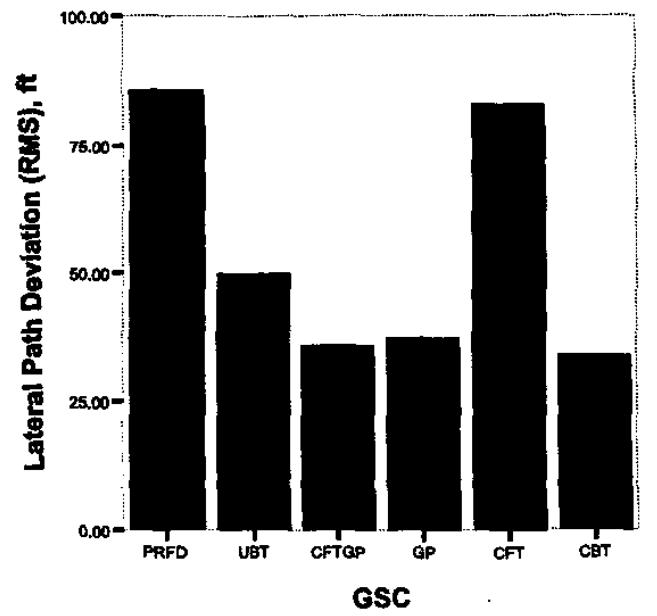

Figure 13. Mean RMS of Lateral Path Deviation

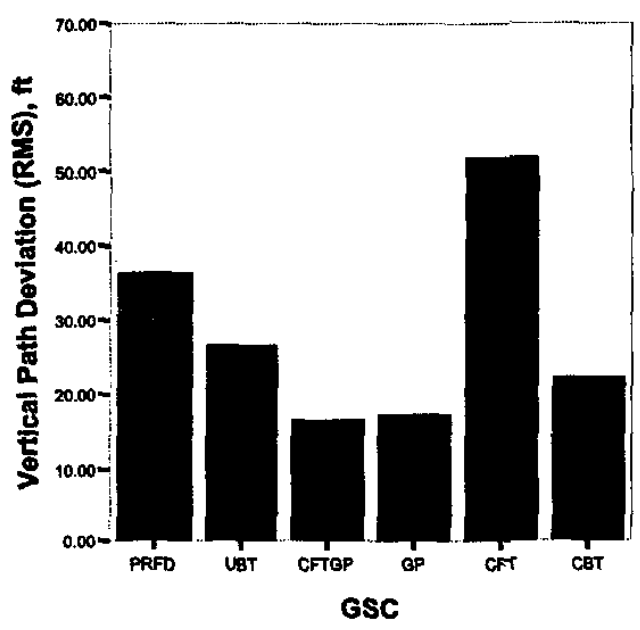

Figure 14. Mean RMS of Vertical Path Deviation

SA results are presented in Figure 15.

ANOVA results, $F(5,720)=68.84(p<.05)$, indicate that the CFT and PRFD belonged to the first subset with lowest perceived situation awareness which is consistent with the above FTE results. However, GP belongs to the $2^{\text {nd }}$ subset, CFTGP to the $3^{\text {rd }}$, UBT to the $4^{\text {th }}$, and CBT to the last subset, with the values of the last subset almost six times as large as the first one, $\sim 15$ versus $\sim 87$, respectively. It can be seen from figure 15 that in general GSCs with tunnel provided increased SA. The primary exception to that trend was the CFT. Path preview, as provided by the more visually complex tunnels, was a main reason cited for the SA results. 


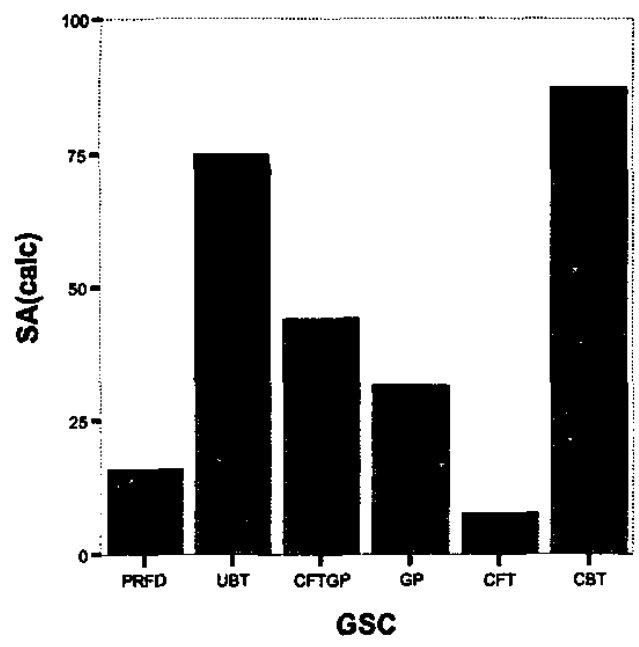

Figure 15. Mean of Situation Awareness, SART

The effect of GSC on TLX was statistically significant TLX, $F(5,720)=37.22(p<.05)$. Three subgroups were formed from post-hoc analysis. The TLX data indicate that CBT and UBT produced the lowest perceived workload, CFTGP and GP produced next higher workload, and the CFT and PRFD created the highest workload (Figure 16). This is somewhat consistent with the results of the SA as PRFD and CFT provided lowest SA and UBT and CBT provided the highest SA. Even though a head tracker was not used in this experiment, based on above results and pilot comments recorded, one can conclude that the corners of CFT were perceived as multiple cues. EPs had to create a mental picture of a tunnel from the crow feet corners. Similar to split cue PRFD, the crow feet required EP's increased attention (low SGC SA) and also increased scanning effort (high GSC workload). The contribution of the ghost plane in CFTGP, as single cue guidance, elevated the standing of CFTGP to the second subset with GP, for both SA and TLX.

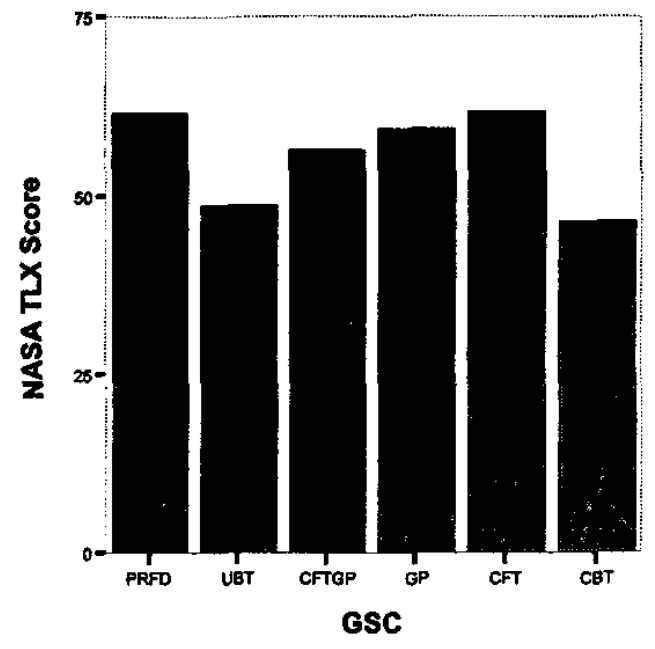

Figure 16. Mean Workload Measure, TLX

\section{Effect of EP Qualifications}

An ANOVA performed on FTEs, SA, and TLX for EP Qualification (Rating) showed statistically significant results for $\operatorname{LPD} F(2,720)=$ $4.64(\mathrm{p}<.05) ; \operatorname{VPD}[\mathrm{F}(2,720)=3.07(\mathrm{p}<.05)]$; and SA F $(2,720)=6.63(p<.05)$ but not for TLX. Furthermore, statistically significant interactions were found between EP Rating and GSC for dependent measures LPD, VPD, and SA. These interactions are discussed in the following section.

\section{Independent Variables Interactions}

Careful study of all interactions showed that there were no statistically significant interactions between TPC and GSCs for the independent variables considered above except between GSC and EP Rating.

The significant interaction between GSC and pilot Rating for LDP was $F(10,720)=2.034$ $(p<.05)$; for VDP $F(10,720)=3.37(p<.05)$; and for SA $F(10,720)=1.8(p<.06)$, (see Figures $17-19)$. 


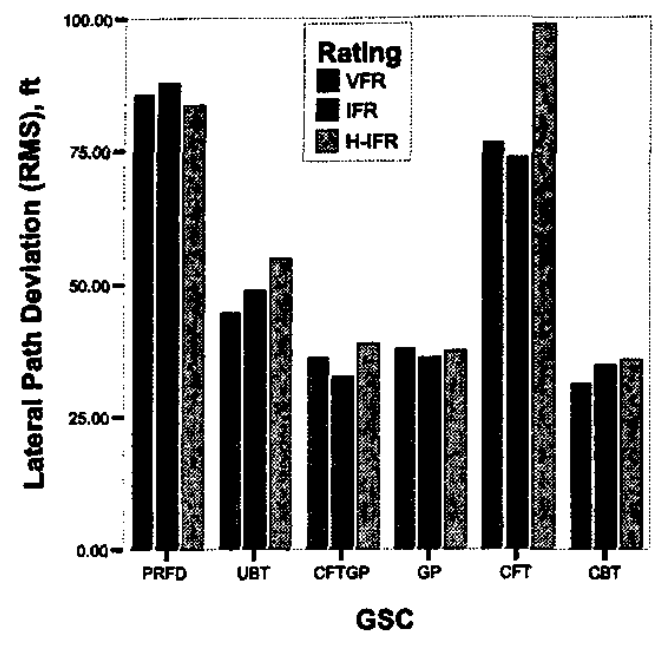

Figure 17. Mean of Lateral Path Deviation

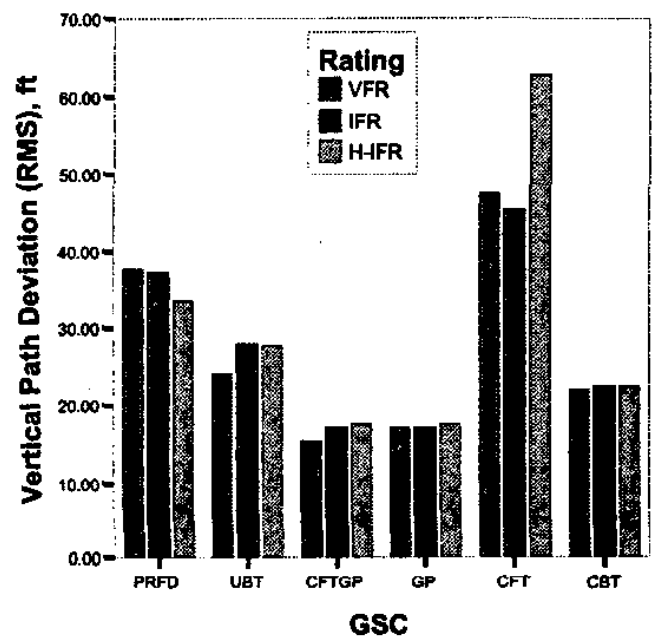

Figure 18. Mean of Vertical Path Deviation

As shown above, HIFR pilots performed somewhat worse than the other two pilot groups for four of the six GSCs (i.e. GP, CFTGP, CFT, and CBT). This could be attributed to the effects of the small display size combined with the average age of the HIFR pilot group (56 years) as compared to the IFR group (38 years). This effect was most evident for the CFT which was the most challenging GSC even for the other two pilot groups. In addition, information provided by the visual integration of SVS terrain with advanced guidance symbology, seemed to be easier to assimilate for the lower-time pilots.

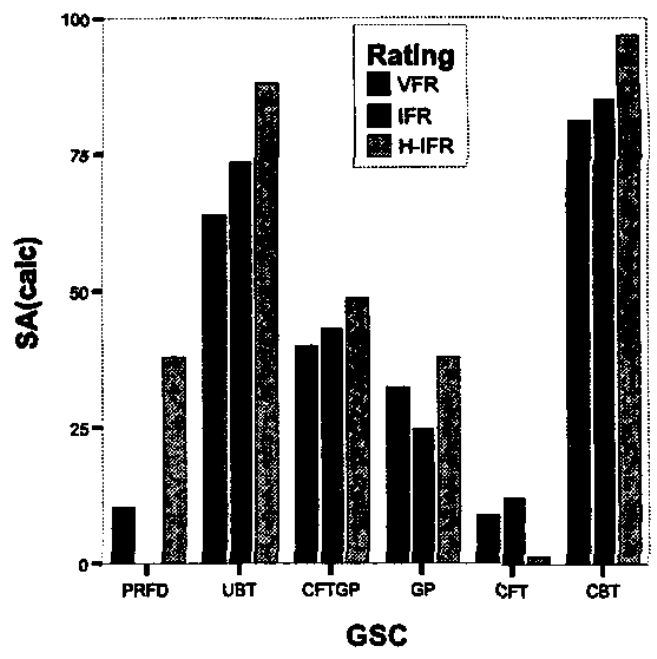

Figure 19. Mean of Vertical Path Deviation

\section{Conclusions}

A fixed based simulation of four terrain portrayals concepts and six symbology concepts on a primary flight display was conducted by three groups of evaluation pilots. The scenario was a challenging mountain pass maneuver. A total of 72 combinations of the above independent variables were flown twice (randomly) by each evaluation pilot.

The effect of GSC and TPC was statistically significant for both lateral and vertical path deviations. EBG produced the lowest VPD. PR and EBG produced the lowest LPD. These results can be attributed to the effective integration of terrain information, as intuitively provided by the EBG and PR concepts. Furthermore, the EBG concept may provide terrain data in an easier to assimilate format than other TPCs, leading to improved VPD results. These results are different from previous experiments with SVS terrain, which indicate only an improvement in SA, and are likely due to the extreme presence of terrain with the evaluation task (i.e. maintain $500 \mathrm{ft}$ AGL in a mountain pass).

Superior FTE was achieved by the use of CFTGP, CBT, and GP (pathway based) GSCs compared to the baseline PRFD. The CFTGP, GP, and CBT concepts reduced FTE by approximately $50 \%$ compared to the PRFD. This demonstrates the enhanced pilot control provided by these GSCs. 
The UBT improved FTE over the PRFD, but was not significantly different from the PRFD for VPD. The CFT performed statistically worse than the PRFD for VPD. This was due to the minimal presence of CFT concept.

There were no statistically significant interactions found between TPC and GSCs.

Qualitative measures of pilot SA and workload followed the quantitative results. The CBT, CFTGP, GP, and UBT all provided significantly superior SA and reduced workload. A major impact of the TPCs was on pilot SA. Higher SA was observed for HIFR pilots as compared to other two groups. The level of SA provided by CCFN was statistically higher than BSBG but less than EBG and PR TPCs. This relationship was also evident by perceived workload (TLX).

In general, all pilot groups performed similarly well when using CFTGP, GP, CBT and UBT, with VPD less than $30 \mathrm{ft}$ and LPD less than $50 \mathrm{ft}$. With the same SVS training provided to all three groups, low time VFR pilots performed as well as IFR pilots in low altitude en-route scenario with IMC. The lower FTE and increased SA achieved for SVS displays, as compared to the baseline PRFD/BSBG, demonstrates the potential and the effectiveness of SVS displays for advanced IMC operations.

\section{References}

[1] T. Abbott, M. Nataupsky and G. Steinmetz, 1987, "Subjective, Physiological and Performance Measures of Eight Primary Flight Displays," Proceedings of OSU APS, 721-727.

[2] G.Sachs, K. Dobler, P. Hermle, 1998, "Synthetic Vision Flight Tests for Curved Approach and Landing," TU Muenchen, IEEE 0-7803-5086-3.

[3] M.P.Snow and J.M. Reising, 1999, "Effect of Pathway-in-the-Sky and Synthetic Terrain Imagery on Situation Awareness in a Simulated Low-Level Ingress Scenario," Wright-Patterson AFB, ASC-99. 1229.
[4] Y.A. van Houten, 1999, "Attentional Effects of Superimposing Flight Instrument and Tunnel-inthe-Sky Symbology on the World," National Luchten Ruimtevaartlaboratorium, NLR-TP-99141.

[6] K.W. Williams, 2000, "Impact of Aviation Highway-In-The-Sky Displays on Pilot Situation Awareness," FAA CAMI, DOT/FAA/AM-00/3

[7] Glaab, L.J, M.A. Takallu, 2002, "Preliminary Effect of Synthetic Vision Systems Displays to Reduce Low-Visibility Loss of Control and Controlled Flight Into Terrain Accidents," SAE 2002-01-1550.

[8] Takallu, M.A, Wong, D.T. and M.D. Uenking, 2002, 'Synthetic Vision Systems in GA CockpitEvaluation of Basic Maneuvers Performed by Low Time GA Pilots during Transition from VMC to IMC,' International Advanced Avionics Technology Conference, Anchorage, AK.

[9] Croom, T.L., 2004, “2004 Interactive SVS GA Workshop," Proceedings of the NASA/FAA/GAMA SVS GA workshop, NASA LaRC, http://avsp.larc.nasa.gov/images_svs_GAFAA WS04.html

[10] Hughes, Monica F; M. A. Takallu, 2002, "Terrain Portrayal for Head-Down Displays Experiment," International Advanced Avionics Technology Conference, Anchorage, AK.

[11] Hughes, Monica F.; Louis J. Glaab, 2003, "Terrain Portrayal for Head-Down Displays Simulation Results," 22nd Digital Avionics Systems Conference, Indianapolis, IN.

[12] Glaab, Louis J.; Monica F. Hughes, 2003, "Terrain Portrayal for Head-Down Displays Flight Test," 22nd Digital Avionics Systems Conference, Indianapolis, $\mathbf{I N}$.

[13] D.T. Wong; M.A. Takallu; M.F. Hughes, L.G. Glaab, 2004, "Simulation Experiment for Developing the Symbology for the Approach and TOGA Phases of Flight of Head-Down Synthetic Vision Systems Displays," AIAA Modeling and Simulation Technologies Conference, Providence, Rhode Island. 